\section{Campagne nationale de prévention contre la grippe}

Chaque année, entre 100000 et 300000 patients consultent leur médecin pour des symptômes grippaux, une hospitalisation s'avère nécessaire dans 1000 à 5000 cas et on compte entre 400 et 1000 décès des suites d'une grippe.

Mt. Il s'agit là de chiffres extrapolés pour l'ensemble de la population helvétique sur la base du système de déclaration Sentinella. Ils démontrent clairement que la grippe est une maladie à prendre au sérieux et que les moyens de prévention efficaces dont nous disposons devraient être mieux utilisés. En Suisse, le taux de vaccination contre la grippe est, en effet, plus bas que dans les pays voisins. En France, par exemple, le taux de vaccination chez les personnes retraitées avoisine, depuis des années, les $80 \%$. Afin de rattraper le retard, l'Office fédéral de la santé publique (OFSP) a lancé, pour la première fois, une campagne nationale d'information et de prévention contre la grippe sur plusieurs années.

Lors de la conférence de presse donnée le 12 septembre dernier, le Prof. Thomas Zeltner, directeur de l'OFSP, a présenté les fondements médicaux et le matériel d'information de la campagne. Les personnes âgées et les malades chroniques sont les premiers à tirer un bénéfice du vaccin administré par voie parentérale, vaccin dont la composition doit être changée chaque année selon les recommandations de l'Organisation mondiale de la santé (OMS). Le vaccin pour la saison grippale 2001/2002 comprend des particules virales de trois souches différentes (tab. 1). Le Prof. Zeltner a tenu à préciser que ce vaccin est à disposition en quantités suffisantes pour cette année et que le problème de pénurie de l'année passée ne devrait pas se reproduire. Cela dit, la vaccination contre la grippe ne rencontre pas partout le même accueil dans la population, phénomène qui s'explique notamment par les différences culturelles. Ainsi, force est de constater, en Suisse alémanique, un taux de vaccination (46\%) inférieur à celui de la Suisse romande (53\%). Lors de l'élaboration des moyens d'information de la campagne (affiches, brochures, etc.), il a été tenu compte de ce facteur. Par ailleurs, une brochure un peu plus détaillée a été conçue spécialement pour les médecins.

La Fédération des médecins suisses - représentée à la conférence de presse par le Dr Pierre Bonfils, délégué de la FMH - s'est associée à l'effort d'information et de sensibilisation de l'OFSP afin que la campagne ne touche pas seulement les patients et la population, mais aussi les médecins et le personnel médical.

Le corps médical et le personnel soignant constituent l'un des publics cibles de la campagne de cette année, pour la bonne raison que c'est à eux qu'il re-
Documents (en français, allemand et italien)

- 1 brochure pour médecin et 1 flyer pour patient ont été adressés à 18500 médecins

- 1 carton avec la documentation de la campagne (1 brochure pour médecin, 40 brochures pour patients et 2 posters) a été envoyé directement aux 10000 médecins internistes, généralistes et spécialistes traitant les patients à risques.

Autres commandes gratuites: Centre de documentation sur la prévention de la grippe, case postale, 3000 Berne 6, tél. 03135214 60, fax 03135214 71, e-mail: grippe@bag.admin.ch

vient d'évaluer les indications et contre-indications de la vaccination et de conseiller, sur une base individuelle, les patients et surtout les patients à risque. Les médecins jouent un rôle primordial dans l'atteinte des objectifs de la campagne, à savoir: d'ici à 2005, augmenter de $48 \%$ à $60 \%$ la couverture vaccinale chez les personnes de plus de 65 ans et atteindre un taux de vaccination de $70 \%$ au sein du corps médical et du personnel soignant. Il faut savoir en effet que, chez les personnes âgées, la grippe peut considérablement affecter la qualité de vie, voire mener à la mort. Par ailleurs, les soignants peuvent mettre en danger leurs patients et déclencher de petites épidémies par contamination.

Un article plus fouillé sur la question, rédigé pour la Commission d'épidémiologie de la Société suisse d'infectiologie par S. Zimmerli et K. Mühlemann, vient de paraître dans le Bulletin des médecins suisses [2]. D'autres informations sont disponibles sur un site web (www.grippe.admin.ch) et dans le bulletin de l'OFSP durant la période de vaccination conseillée, à savoir d'octobre à novembre.

Reste encore à signaler que le Nasalflu ${ }^{\circledR}$, vaccin administré par voie nasale, vient d'être retiré de la procédure d'admission par son fabricant, la firme Berna Biotech SA. Il ne sera donc pas disponible pour la campagne de vaccination de cette année. La raison invoquée est l'existence possible d'une relation entre ce vaccin et l'observation de quelques cas de paralysie faciale. Les études mises en route pour élucider cette question n'ont pas encore abouti; les résultats sont attendus avec impatience.

\section{Tableau 1}

Les vaccins recommandés pour la saison hivernale 2001-2002 (hémisphère nord) contiennent les souches suivantes [1].

un virus analogue à A/New Caledonia/20/99(H1N1)

un virus analogue à $\mathrm{A} / \mathrm{Moscow} / 10 / 99(\mathrm{H} 3 \mathrm{~N} 2)$

un virus analogue à B/Sichuan/379/99

\section{Références}

1 OMS. Composition recommandée des vaccins antigrippaux pour la saison 2001-2002. Relevé épidémiologique hebdomadaire 2001;76(8):58-61.

2 Zimmerli S, Mühlemann K. La prévention de la grippe aujourd'hui. Bull Med Suisse 2001;82(39):2071-6. 